\title{
Mental Health and Early Adolsecent: A Prevalence Study
}

Karimah Hanim Abd Aziz ${ }^{1}$, Nurjasmine Aida Jamani ${ }^{2}$, Nur Wal Affa Aziz ${ }^{3}$, Fatin Shahira Hamdan ${ }^{3}$

${ }^{1}$ Department of Community Medicine, Kulliyyah of Medicine, International Islamic University Malaysia

${ }^{2}$ Department of Family Medicine, Kulliyyah of Medicine, International Islamic University Malaysia

${ }^{3}$ Kulliyyah of Medicine, International Islamic University Malaysia

Presenter: Solehuddeen Shaatibi Zahari

Introduction: Prevalence of mental health problems among children and adolescent is increasing in trend as reported in the National Health Morbidity Survey (NHMS). Male, younger age group and from low socioeconomic status were at higher risk to develop this problem. This study aimed to measure the prevalence of mental health problems and their associated factors among young adolescent in Kuantan, Pahang. Materials and Methods: A cross-sectional study was conducted among 211 standard 4 and 5 (aged 10-11) students in July 2017. A validated Malay version Strength and Difficulty Questionnaire (SDQ) which consisted of five problem subdomains (emotional, conduct, hyperactivity, peer and prosocial) was filled by parents. The data were analysed using descriptive statistics and logistic regression. Results: The prevalence of mental health problems among young adolescents was $16.3 \%$. The commonest mental health problems were peer (45.8\%), conduct $(27.2 \%)$ and prosocial $(18.7 \%)$. Higher household income (OR: 2.66, Cl: 1.186-5.995), higher household number (OR: 0.593, Cl: 0.416-0.845) and no family history of mental illness (OR: 24.53, Cl: 1.506-399.65) were associated with mental health problems. Conclusion: We found that the prevalence of mental health problems was higher than that was reported by NHMS in 2015. Adolescents from higher household income family and no family history of mental problems were more likely to develop emotional problems. However, higher household number was found to be a protective factor against mental health problems. 